\title{
The surgical treatment of ulcerative colitis
}

\author{
J. C. Goligher \\ Professor of Surgery, University of Leeds
}

Time was when the surgery of ulcerative colitis was in the highest degree controversial and the surgeon had to fight-and fight hard-for a share in the management of this disease, which naturally lent a certain evangelical fervour to his writings and utterances on the subject. But that struggle has been largely won, surgery now has an established place in the treatment of this condition, and the whole plan of surgical therapy has become much more stereotyped.

However, there are still a few debatable issues left, and it may be as well to commence with one of these outstanding problems-and, indeed, to choose the point of greatest controversy remaining in the surgery of colitis. The standard surgical treatment for diffuse ulcerative colitis at the present day is colectomy, but does this also invariably mean rectal excision and ileostomy, or not?

The majority of surgeons would reply most emphatically that it does, but a very few, including notably Aylett (1964), would claim that equally good results can usually be obtained by a colectomy and ileorectal anastomosis, which preserves the rectum and avoids an ileostomy. On the face of it this would seem hard to accept, for diffuse ulcerative colitis is really a proctocolitis, which invariably involves the rectum as well as the colon, so that colectomy and ileorectal anastomosis means retaining and using a diseased rectal stump. On theoretical grounds this must be regarded as a very illogical way of practising excisional surgery for diffuse colitisone indeed that would seem almost designed to facilitate persistence or recurrence of the disease or the development of complications in the remnant of rectum.

Of course what matters in Medicine, as in most other affairs in this world, is not what should happen but what actually does happen, and the results reported by Aylett (1964) have been most impressive-some 300 cases treated by this method with roughly $90 \%$ of satisfied patients. As a consequence, a number of other surgeons have felt compelled-almost against their better judgment-to give this operation a trial in diffuse colitis, but no one has been able to reproduce quite the achievements claimed by Aylett. Indeed in most series treated by colectomy and ileorectal anastomosisas in my own small group of cases (Goligher, 1967), and in the series reported by Griffen, Lillehei \& Wangensteen (1963), Ault (1960), Muir (1959), and by Anderson (1960) - this operation can be considered to have failed in up to $50 \%$ of the patients. Even Turnbull (personal communication 1966) and Hughes \& Russell (1967), who have both been enthusiastic and regular users of this operation, have reported $20-30 \%$ of bad results-and Turnbull tells me that he fully expects the long-term failure rate to be of the order of $50 \%$.

It must be admitted that it is not easy te define exactly what constitutes a failure in thi context. Most patients with diffuse colitis improve remarkably in their general health-usually u\& to full normal general health-after a colec $§$ tomy and ileorectal anastomosis. Consequently even if they should continue to have pretty sever changes in the rectal stump and to experience quite bad diarrhoea with up to ten or twelve motions a day, even with some blood and mucus, they may none the less be so pleased with their overall condition that they are prepared to put up with this state of affairs indefinitely rather than have an ileostomy, against which they have usually been warned so vehemently before operation. I have now had to convert some twenty-five patients to ileostomies following unsuccessful colectomies and ileorectal anastomoses for diffuse colitis (a few of whom had been originally operated on by me, the majority by other surgeons). Without exception the result has been quite a revelation to them, for they have found the ileostomy to be a blessed relief from their incessant diarrhoea, and have bitterly regretted not having resorted to it sooner.

Some surgeons have proposed that most patients with diffuse colitis coming to operation should be given a chance with colectomy and ileorectal anastomosis in the first instance, on the grounds that if they fail to do well with it, they can always be converted subsequently to ileostomy. This naïve suggestion overlooks three 
major difficulties. First of all, it glosses over the fact that a second operation of this kind may be technically quite difficult, is inevitably associated with some contamination, and is often followed by an unpleasant degree of sepsis. Secondly, it ignores the difficulty already mentioned of inducing patients with poor results after ileorectal anastomosis to accept a further operation involving the creation of an ileostomy. And, thirdly, if the disease persists in the rectal stump and the patient refuses to have a second operation, it exposes him to the undoubted risk of developing a carcinoma in the chronically inflamed rectal mucosa. I would like to emphasize to you that a number of such growths after colectomy and ileorectal anastomoses for diffuse colitis have now been reported in the literature, and $I$ am sure we may expect more in the fullness of time.

Altogether, the experiences outlined above have convinced nearly all surgeons that colectomy and ileorectal anastomosis is in general an unsatisfactory operation for diffuse ulcerative colitis with rectal involvement,* and that for patients requiring surgical treatment for this condition the only reliable operation is a complete excision of the large intestine in one or two stages, combined with a permanent ileostomy.

\section{The ileostomy and its after-care}

Fortunately there have been significant advances in the technique of ileostomy and its aftercare, which have gone a long way towards robbing this form of artificial anus of its more objectionable features. On the technical side the important things are: firstly, to site the stoma in a position where it can be accurately and securely fitted with a modern ileostomy bag; secondly, to make the end of bowel project adequately so that it protrudes well into the appliance ; and thirdly, to construct the actual opening in such a way that it is unlikely to undergo subsequent fibrosis and stenosis, or undue protrusion or recession.

As regards siting (Fig. 1) opinions differ as to the best place to put the ileostomy-high up in the waist line or lower down in the R.I.F.but I think the essential considerations about its position are: (a) that it should not be made in the line of the main laparotomy incision, but

\footnotetext{
*It is, however, a very suitable procedure for cases of so-called right-sided or segmental colitis sparing the rectum and a variable amount of the distal colon, but such a distribution of disease in true ulcerative colitis is excessively rare-some would say it never occurs-though it is quite common in Crohn's disease of the large bowel, when it also lends itself well to treatment by colectomy and ileorectal anastomosis.
}

should be established at a separate trephine wound surrounded by smooth, flat, unscarred skin ; and (b) that it should not be too close to the umbilicus or the anterior superior iliac spine. The most secure place is probably in the waist line as shown on this diagram, but women patients object strongly to this position as ruinous to their figure if they are wearing a tight fitting dress, and if a man uses a belt instead of braces, as is becoming more popular in this country now, he will also want the stoma not in the waist line but a bit lower down.

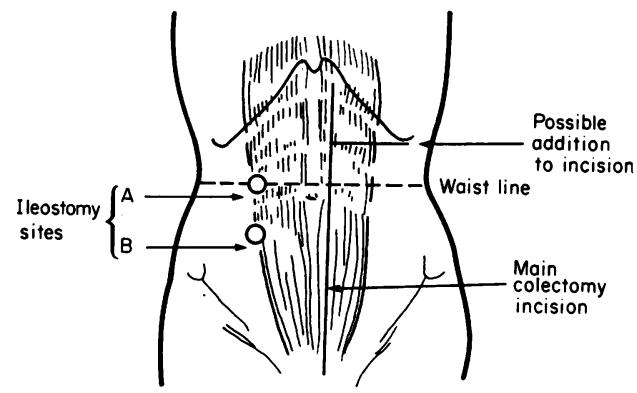

FIG. 1. Diagram showing main incision used for primary colectomy or proctocolectomy and the sites favoured for the ileostomy. (From Goligher, 1967.)

The actual technique of ileostomy construction now generally used is that of eversion and primary suture and Fig. 2 shows the sort of soft supple ileostomy spout which this method produces, projecting $1 \frac{1}{4}-1 \frac{1}{2}$ in. or so, and surrounded by smooth flat skin, ideal for taking a modern adherent ileostomy bag. Fig. 3(a) illustrates such an appliance. There are several other patterns available as well. The special feature of this one is that the more rigid part surrounding the hole in the back, into which the ileostomy bud pro-

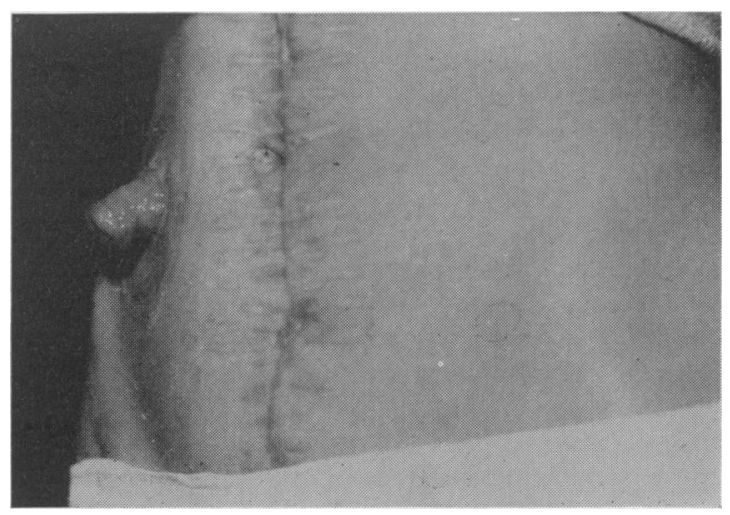

FIG. 2. Photograph showing a well sited and perfectly formed ileostomy. 
trudes, can be separated. The plan of use is to stick this semi-rigid part, known as the flange, on to the skin around the ileostomy with a double-sided adhesive plaster, or cement (Fig. 4a). The gap or moat between the ileostomy bud and the wall of the flange is filled with a cigarette of cotton wool (Fig. 4b) to avoid accumulation of faeces in this crevice. The hole on the back of the bag proper is then stretched to fit on to a groove near the edge of the flange (Fig. 4c and d). Normally the flange remains in position for a week or so at a time, the bag is changed on the flange daily, and the contents of the bag are emptied three or four times a day.
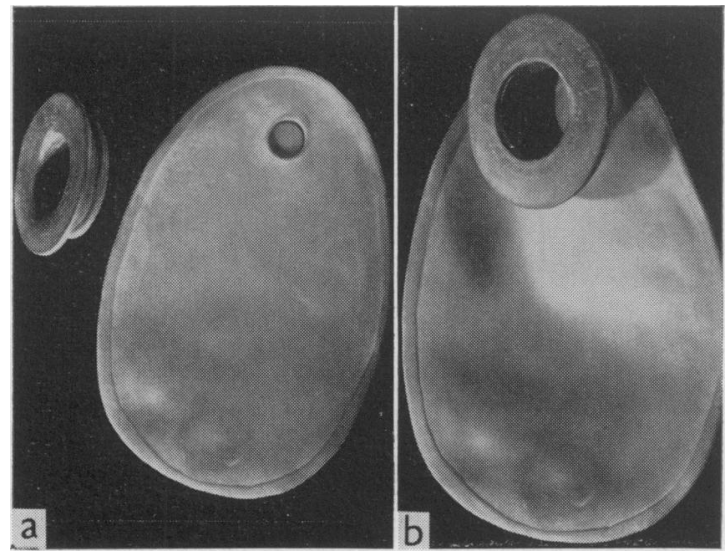

FIG. 3. A two-piece rubber ileostomy appliance: (a) flange and bag separate; (b) both pieces fitted together. (From Goligher, 1967.)

Rarely, if some degree of soreness of the periileostomy skin should develop and it is difficult to make the plasters stick, a valuable alternative adhesive is Karaya Gum Powder, which forms a glue fixing the flange and also soothing the inflamed skin to which it is stuck. Usually this is successful over a period of 3 or 4 weeks in clearing up the soreness.

In all but a very small minority of cases the use of an adherent bag ensures for the patient a completely water-tight connection between the appliance and the abdominal wall which avoids all soiling and permits a really full existence which may include a wide range of activities. Thus most patients are able to return to their previous employment even if this were a heavy manual job, always provided that it doesn't expose the stoma itself to injurious pressure, as in the crawling manoeuvres of mining, or the lifting of cases and packages resting partly on the abdomen. Similarly recreations and sports of most kinds are possible, consistent with the
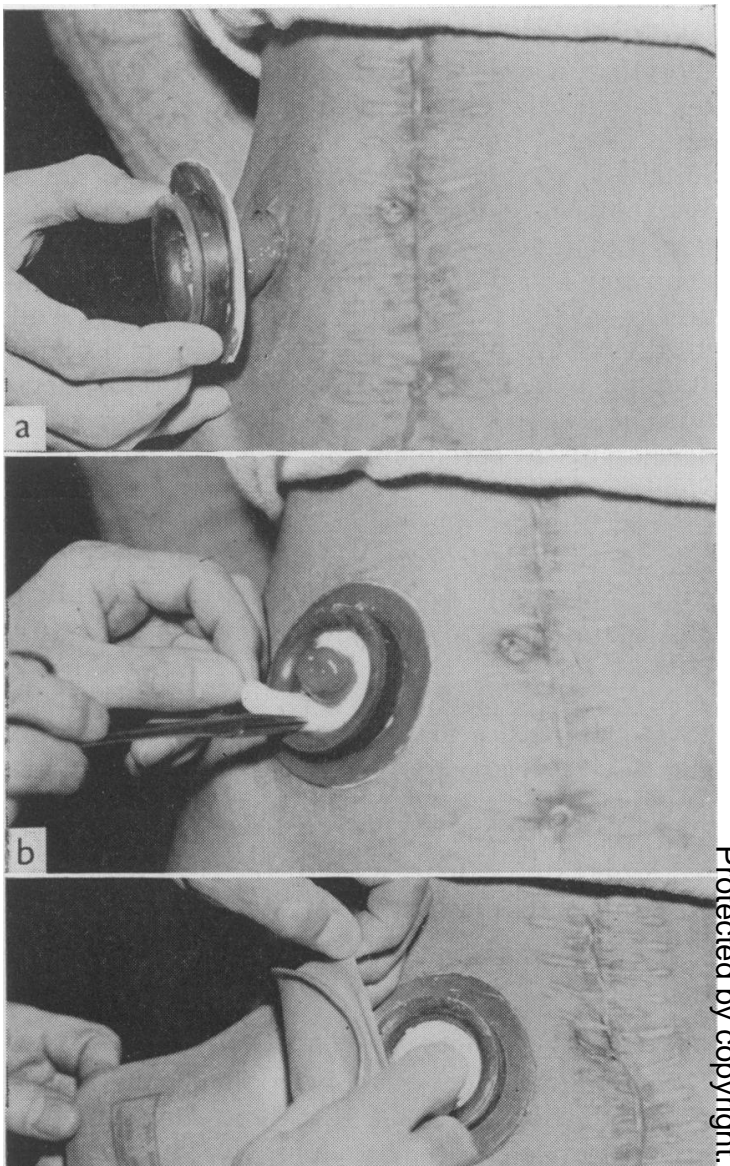

Fig. 4. Application of two-piece ileostomy bag: (a) the flange, to the deep aspect of which has been stuck a double-sided disc of adhesive strapping, is being applied to the skin around the projecting stoma; (b) the moat between the wall of the flange and the ileostomy bud is being filled in with a 'cigarette' of cotton-wool; (c) the hole on the back of the bag proper is being stretched, and fitted on to a groove near the free edge of the flange; (d) bag completely fitted to flange. 
patient's age and inclinations-such as dancing, golf, tennis and even swimming with a thickfronted swim-suit. But obviously so-called body contact games and athletics, such as rugger and wrestling, are unsuitable as likely to injure the ileostomy or displace the appliance.

The patient's great difficulty often is to believe that all this will hold for her if she accepts operation, for, when first contemplated, ileostomy cannot fail to appear a revoltingly unnatural arrangement to a lay person, particularly to a young woman who sees in it the complete disintegration of all her romantic aspirations, or even the destruction of her happily established married life. In overcoming her apprehension nothing is more helpful than for her to have a heart-to-heart talk with another well-adjusted ileostomy patient of the same sort of age and from the same walk of life, and the Ileostomy Association is helpful in providing such confidantes, if you don't have a large clientele of your own on which to call. It is seldom indeed that this plan proves unsuccessful, and also very rarely that a patient who has had severe colitis treated by operation regrets in retrospect the decision to resort to ileostomy. As for sex life, so far from ileostomy being a bar it seems to provide a positive stimulus, presumably because of the great improvement in general health, and very many women ileostomists have apparently found no difficulty at all in getting engaged and married and bearing children (usually per vias naturales).

Actually in this connection male ileostomists very occasionally fare less well, in that in a small proportion of them after protocolectomy there is impairment of sexual function (Goligher, 1967). It is difficult to decide whether this is entirely physical, due to injury to the pelvic autonomic nerves at operation, or psychological, for it is a common experience that such disorders are restricted-fortunately perhaps-largely to older patients. But sexual dysfunction does rarely occur in young men as well, so that the disturbance is definitely sometimes due to nerve injury. It is unnecessary to emphasize what a tragedy such a complication can be to a young man and how important it is to exercise the utmost vigilance at operation to protect the sympathetic and parasympathetic nerves from injury, by defining and carefully preserving the presacral nerve when tying the inferior mesenteric vessels and also by keeping as close as possible to the rectum during the pelvic dissection. As an additional precaution one could certainly argue a good case when dealing with young male colitics, for temporarily leaving the rectum in the first instance by performing only a subtotal colectomy rather than a proctocolectomy as the initial operation, and removing the rectal remnant some years later after he has had a chance to produce a family. However, this is only a partial solution to the problem, for whenever the rectum is removed the risk of nerve injury and subsequent sexual disorder exists, and there is certainly more to sex life than just the production of children. But unless the rectum is eventually excised the patient is exposed to a gradually increasing danger of developing a colitis carcinoma in it as the years roll by.

\section{Indications for surgical treatment}

\section{Elective operation}

Intractability and chronic invalidism. These are quite the commonest reasons for resorting to operation. In some cases the indications on these grounds are only too obvious, as when a patient with a history of severe relapsing colitis for several years presents in a state of extreme emaciation, almost of Belsen or Auschwitz type, with gross changes in the large bowel and often complications such as an anal fistula, arthritis or pyoderma gangrenosum. Even the most conservatively-minded and reactionary of physicians would, nowadays admit that the only chance of restoring such an individual to good health and a worthwhile existence lies with surgery, the result of which may be most dramatic, with the patient often doubling or even trebling her weight within a year or two of proctocolectomy-which is not quite so astonishing as it sounds when it is borne in mind that the weight at the time of operation may be as little as $4 \frac{1}{2}$ stones.

In certain other cases surgical treatment is just as clearly contraindicated. These are patients with mild distal forms of the disease confined to the rectum and possibly the distal sigmoid, who, remarkably enough, usually present first to the surgeon in his Rectal Clinic, because constitutional symptoms are generally negligible and the main complaint is of slight bleeding, suggestive of haemorrhoids. As a rule no question of surgery arises for such cases, except occasionally in elderly patients who may be profoundly ill even with a distal proctosigmoiditis and may require elective or emergency radical operation for it. It should be remembered also that in cases starting off as a proctitis, the pathological changes may subsequently-perhaps in a period of yearsgradually extend proximally to produce a total or near-total colitis, for which surgical treatment may well be required. If the clinician is not to slip up in his management of this disease it is essen- 
tial for him to bear in mind the important fact that colitis is a dynamic condition constantly liable to change its extent in this way.

In between these two extremes of very severe and very mild disease, there is a wide spectrum of patients with diffuse colitis, of varying degrees of severity and extent of involvement of the bowel, associated with more or less diarrhoea and constitutional disturbance, for whom the verdict for or against surgery has often in the past been a very fine one indeed. One of the difficulties hitherto encountered in reaching a decision in these cases has been the lack of really reliable information regarding the outcome of continued conservative treatment, against which to weigh the better known risks of surgical measures. However this defect in our knowledge has been largely remedied in the last 3 or 4 years by the very careful follow-up studies of Edwards \& Truelove (1963) in Oxford, and of our own group in Leeds (Watts et al., 1966a, b). These surveys provide no grounds whatsoever for complacency on the party of the physician. They show that following an initial attack of colitis, and often despite maintenance therapy with steroids or sulphasalazine, patients usually have further attacks. These may be mild, moderate or severe, and the patients most likely to develop severe attacks, which medical management often fails to arrest, resulting in resort to urgent surgery with all the risks that this entails, are those with total or near-total involvement of the large bowel, and those over 60 years of age. Watts et al. (1966b) found that the average mortality per year of follow-up on conservative treatment in patients whose colon is totally involved is $2.7 \%$, and, in patients over 60 years, $4.9 \%$; even though in many of these older patients the disease is confined to the distal large bowel. Actually the annual mortality in patients over 60 , who also have total involvement, is $11 \cdot 1 \%$.

Accepting the operative mortality of elective proctocolectomy and ileostomy as about 3 or $4 \%$, and allowing that there may be a late mortality over the next 5 or 6 years of 2 or $3 \%$ or more due to intestinal obstruction and other sequelae, the overall figure for the risks of elective operation may be of the order of 5 or $6 \%$. It will be seen from Fig. 5 that it takes on the average only 3 or 4 years of conservative management of cases of colitis with total involvement to exceed this mortality; and the dangers of continued medical treatment in older patients accumulate even more quickly, though admittedly the hazards of elective operation are probably also greater in the higher age groups. Certainly in the light of our findings it would seem advisable to us to recommend strongly to all patients who develop total colitis at any stage of their illness, and to many patients over 60 years of age even with only limited disease, that they should submit to an elective proctocolectomy as soon as possible. They may not immediately accept this advice, especially if they had had only a short history, but if they then have another attack, the truth of one's remarks becomes evident to them and they generally agree to operation.

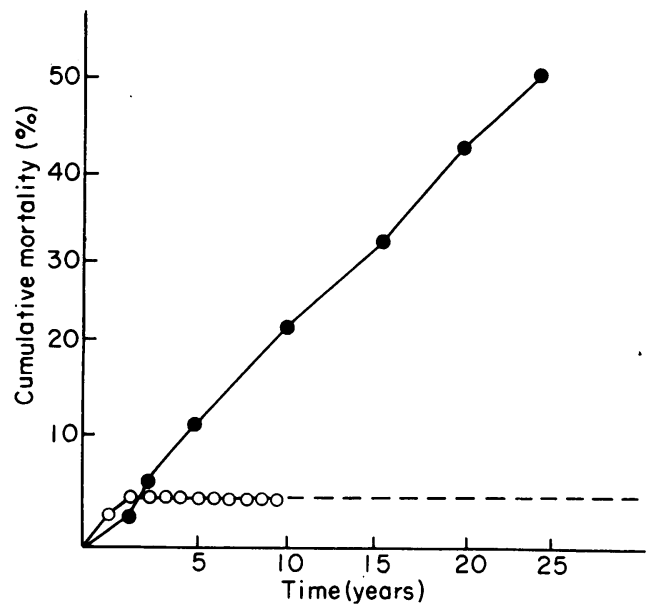

Fig. 5. Diagram illustrating the relative risks of continued conservative treatment (including the mortality of emergency surgical intervention when medical treatment fails to secure remission in severe attacks) and of elective proctocolectomy (immediate and subsequent mortality). Total involvement (present day treatment); $O$, elective surgery (121:cases). (From Goligher, 1967.)

The risk of malignant change. Another reason why moderate or borderline cases should not be left dragging on indefinitely in indifferent health with smouldering colitis is the risk of malignant change. As MacDougall (1964) and de Dombal et al. (1966) have pointed out, this is a complication essentially of total or near-total colitis, which has been present for over 10 years. When colitis carcinoma does occur, it carries a formidable mortality, for it is usually highly active and only rarely curable by excision. Indeed the only satisfactory way of dealing with it surgically is to anticipate its development by prophylactic proctocolectomy in all long standing cases of colitis with total or near-total involvementthough it is to be hoped that all patients with extensive colitis of this kind would now have their colons removed years before the risk of cancer could seriously arise. 
The possibility of impaired development in children and adolescents. In young patients an additional reason for advising operation is the possibility of physical retardation due to continued colitis. One is naturally reluctant to advise ileostomy to a child, but the general experience with colitis in young children is that surgery is often required and that ileostomy is very well accepted as a rule and seems to cause no particular psychological upset.

Local ano-rectal complications. These are seldom an indication per se for radical operation for the underlying bowel condition, but they may provide additional grounds for resorting to surgery in any particular case.

Systemic complications. Arthritis, iritis and pyoderma, etc., if they fail to respond to medical treatment for the colitis, are also a strong indication for proctocolectomy.

\section{Urgent operation}

The striking thing is perhaps how much more hazardous are such interventions undertaken during acute phases of colitis that have failed to remit on medical treatment than are interval operations (Table 1). The mortality of elective operation is now relatively satisfactory at $3 \%$. By contrast the mortality of urgent operations stands pretty high $-19 \%$. In extenuation of these somewhat depressing results it should be emphasized that all the ninety-one patients treated by urgent operations were seriously ill, some of them certainly being in extremis and being what many people would term 'fulminating' cases. No less than sixteen patients had one, or more, perforations of the colon. When dealing with an illness of this overwhelming severity it is difficult to avoid a considerable operative mortality.

\section{TABLE 1}

Operative mortality of radical operations for ulcerative colitis according to the urgency of intervention (author's personal series)

\begin{tabular}{lcc}
\hline & Cases & $\begin{array}{c}\text { Mortality } \\
(\%)\end{array}$ \\
\hline Elective operation & 229 & 3 \\
Urgent or emergency operation & 91 & 19 \\
\hline
\end{tabular}

The question is what can be done to lower the mortality of urgent surgery. Many surgeons have felt that if patients could be brought to operation earlier in the course of their severe attacks the mortality might be substantially reduced. But physicians have naturally been reluctant to invoke surgical aid till the patient has been given a real chance to achieve a spontaneous remission of the acute attack with the aid of steroidschiefly for fear of inflicting an unnecessary ileostomy. However our follow-up survey shows that this fear is largely groundless because the majority of patients who survive a severe acute attack of medical therapy eventually came to elective or emergency ileostomy and proctocolectomy on a subsequent occasion. In the light of this experience we have for the last 3 years been resorting to urgent operation in severe attacks much sooner-within 3 or 4 days of admission if there is no striking response to intensive medical therapy or if a sudden deterioration in general condition takes place-and the results to date show a marked improvement, with a lowering of the operative mortality of severe attacks from $25 \%$ in the years prior to 1963 down to $4 \%$ since that date, though it must be admitted that this result could be partly due to a slight difference in the sort of cases coming to us in the last 2 or 3 years. It is also only fair to add that we have had an exceptionally wide experience with the operative management of acute phases of colitis and this may have enabled us to achieve better results in these difficult cases than would be obtainable by the average surgeon and his team. It is with some diffidence, therefore, that I recommend for general adoption a policy of earlier surgery in these very ill patients with severe attacks of colitis. But I would certainly urge that, if intensive medical treatment in such cases has not been rewarded by any convincing evidence of commencing remission within a week or so, urgent operative intervention should be seriously considered. In addition immediate operation may be required at any stage because of the occurrence of colonic perforation-which, it must be stressed, shows itself clinically in these patients more often by a sudden deterioration in the general condition than by the development of the classical abdominal signs of a perforated viscus. Slightly less urgent an indication for emergency intervention is the appearance of so called acute toxic megacolon (Fig. 6), which has to be sought by regular daily plain $X$-ray examination of the abdomen in these acute cases undergoing medical treatment. Its significance is that it may be a precursor to perforation, besides being often associated with severe toxaemia. Deep undermining ulceration of the colon wall on plain X-ray examination or emergency barium enema study (Fig. 7) has also been regarded by Brooke \& Sampson (1964) as predisposing to perforation, but unquestionably many patients with these gross radiological appearances have surmounted their severe attack on conventional 


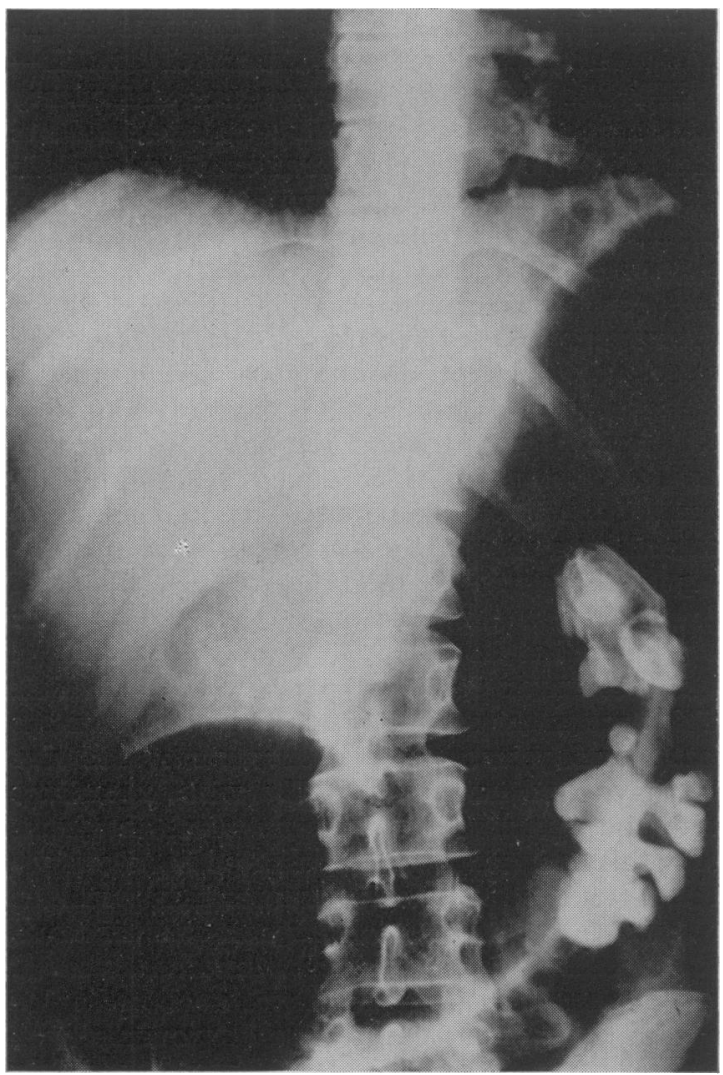

Fig. 6. Plain radiograph of the abdomen in a patient suffering from a severe attack of ulcerative colitis complicated by the development of acute dilatation of the colon (note also the stag-horn calculus in the left kidney). (From Goligher, 1967.)

medical therapy. Massive haemorrhage could be an indication for emergency operation, but almost never is because it usually ceases on conservative management with blood transfusion.

\section{Conclusion}

The fact has to be faced that for patients with extensive and severe ulcerative colitis there is no satisfactory long-term medical treatment that can be relied upon to keep them in good health and avoid further severe attacks of the disease. The best hope for these patients lies with radical surgical treatment, which, despite the magnitude of the intervention and the fact that it involves the creation of a permanent ileostomy, is remarkably well-tolerated as a rule and has provided for many sufferers virtually a new life.

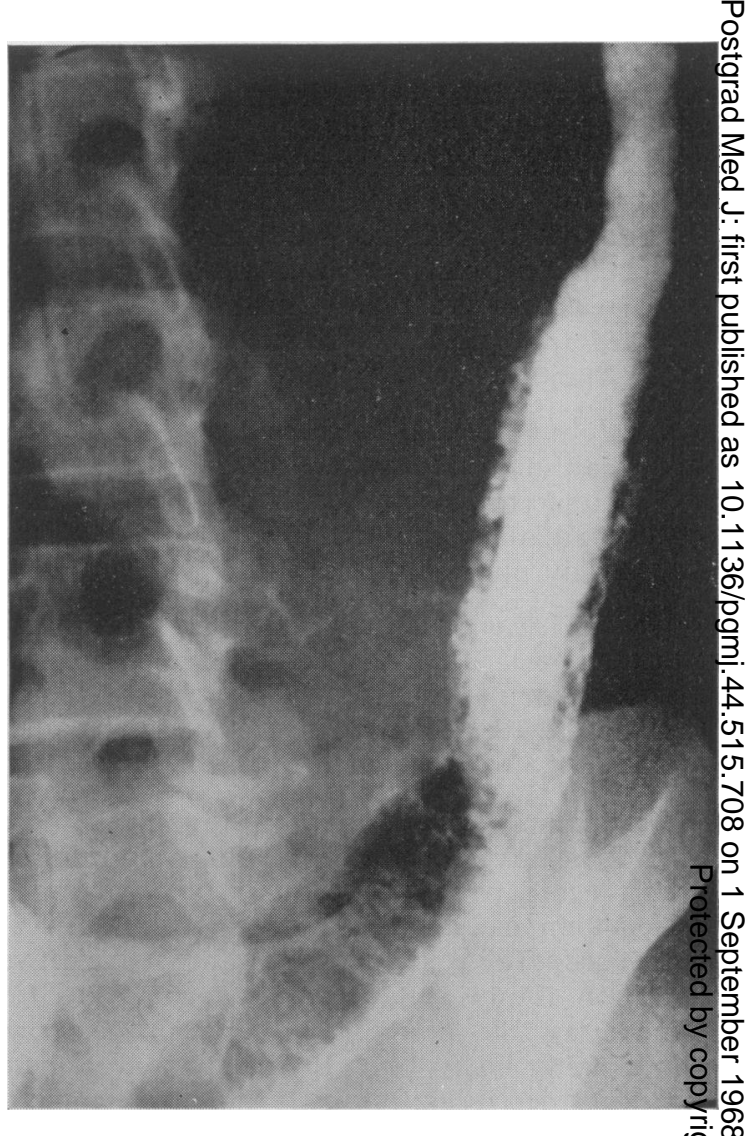

FIG. 7. Urgent barium enema examination in patien with severe attack of ulcerative colitis, showing deep ulceration-the so-called double contour appearance. (From Goligher, 1967.)

\section{References}

Anderson, A.F. (1960) Colectomy and ileorectal anastomosis in surgical treatment of ulcerative colitis. Austr. N.Z. Surg. 30, 107.

Ault, G.W. (1960) Selective surgery for ulcerative colitis. Proc. roy. Soc. Med. 52, 11.

AYLETT, S.O. (1964) Ulcerative colitis treated by total $\delta$ colectomy and ileorectal anastomosis: a ten year review. Proc. roy. Soc. Med. 56, 183.

Brooke, B.N. \& SAMPSON, P. (1964) An indication for $\stackrel{ }{\supset}$ surgery in acute ulcerative colitis. Lancet, ii, 1272.

DE Dombal, F.T., Watts, J.McK., Watkinson, G. \& Goligher, J.C. (1966) Local complications of ulcerative $\mathrm{N}$ colitis: stricture, pseudopolyposis and carcinoma of the $\sigma$ colon and rectum. Brit. med. J. i, 1442.

EDWards, F.C. \& TRUelove, S.C. (1963) The course and prognosis of ulcerative colitis. I. Short-term prognosis. $\omega$ II. Long-term prognosis. Gut, 4, 299.

Goligher, J.C. (1967) Surgery of the Anus, Rectum and Colon, 2nd edn. Bailliere, Tindall \& Cassell, London. 
Griffen, W.O., Lillehei, R.C. \& Wangensteen, O.H. (1963) Ileoproctostomy in ulcerative colitis: long-term follow-up extending in early cases to more than 20 years. Surgery, 53, 705.

Hughes, E.S.R. \& Russell, I.S. (1967) Ileorectal anastomosis for ulcerative colitis. Dis. Colon Rectum, 10, 35.

MacDougall, I.P.M. (1964) The cancer risk in ulcerative colitis. Lancet, ii, 655.
Muir, E.G. (1959) The results of ileo-rectal anastomosis. Anglo-American Conference on Proctology, Proc. roy. Soc. Med. Supplement 1959, 25.

WatTs, J.McK., DE Dombal, F.T., Watkinson, G. \& GoligheR, J.C. (1966a) The early course of ulcerative colitis. Gut, 7, 16.

WatTS, J.McK., DE Dombal, F.T., Watkinson, G. \& Goligher, J.C. (1966b) The long term prognosis of ulcerative colitis. Brit. med. J. i, 1447. 